\title{
Improved Active Frequency Drift Anti-Islanding Detection Method
}

\author{
Ge Yangyang ${ }^{1}$, Junjie Sun ${ }^{3}$, Wang Gang ${ }^{1}$, Gao Zhiqiang ${ }^{2 a}, \mathrm{Yu} \mathrm{Li}^{2}$ \\ ${ }^{1}$ State grid Liaoning province power co.,LTD electric power research institute, Shenyang, China \\ ${ }^{2}$ School of electric power, Shenyang institute of engineering, Shenyang, Liaoning province, China \\ ${ }^{3}$ School of electrical engineering, Wuhan university, Wuhan, Hubei province, China \\ agaozhiqianghit@163.com
}

\begin{abstract}
Keywords: active frequency drift(AFD); islanding detection; photovoltaic(PV); active power; power quality
\end{abstract}

\begin{abstract}
Since passive anti-islanding detection have largely blind spots, easily fluctuation and so on shortcomings, moreover active frequency drift have Some drawbacks too. For example distortion of current and fluctuation of active power injecting into grid associated with islanding detection. a based on variable active power improved active frequency drift anti-islanding detection method is proposed. The technology acquired chopping fraction of active frequency drift through adding variable active power into offset. So as to achieve positive feedback of voltage frequency at the point of common coupling in islanding state and realize anti-islanding function. Under normal circumstances injected power quality into grid has improved greatly, and ensure that the less pollution to the grid. The simulation and experimental results show that the anti-islanding detection methods not only improve detected speed of anti-islanding detection, but also to ensure have better power quality that the injected into the grid
\end{abstract}

\section{Introduction}

With the development of renewable energy sources, more and more distributed energy injects into the grid, especially PV. When the grid fails or when a power maintenance, and PV System systems should be able to promptly disconnect from the grid. When the grid has been disconnected, if PV system can not be detected in time and quickly cutted off, there will be the self-powered that a power company can not contro, which would lead to the islanding ${ }^{[1.2]}$. Islanding is a unique phenomenon at grid system, it will have serious hazards. One is changing the voltage and frequency of users after the power is restored. Another hazard is life safety because the grid does not know the system have or no have power. Therefore, the corresponding international standards, photovoltaic inverter must have anti-islanding detection capability, that is, after the islanding phenomenon occurs, PV systems can be detected within the specified time and promptly disconnect the power grid. There are active and passive two most common methods among the many islanding detection methods ${ }^{[3]}$.

Passive Islanding Detection. Since PV grid system uses current control, voltage and frequency becomes unstable during islanding phenomenon occurs, therefore, it can detect changes of voltage and frequency in local, and determine whether there is an islanding phenomenon ${ }^{[4]}$. According to which reference is different electrical parameters, the passive islanding methods can be divided into following methods:

(1) Over / under voltage, over / under frequency protection method is to set common voltage and frequency of the upper and lower threshold, once the voltage or frequency exceeds the threshold value, it is considered an islanding phenomenon, and should fast cut off from the grid $^{[5,6]}$.

(2) phase transition detection is the system output current with the grid voltage frequency, under normal conditions the phase difference is zero. Once a grid outage, the phase difference will be determined by the load, if the detected phase deviation is relatively large, it is considered that there are an islanding phenomenon. 
(3) harmonic detection is once grid outage, the harmonic content of the voltage waveform at a common node becomes larger, containing a large number of harmonics. Therefore, the detection current harmonic content is determined whether islanding has occurred.

Active Islanding Detection.Passive detection of islanding detection although simple, but there are not easy to set the threshold and detect large blind spots. so active islanding detection method came into being. Active detection system occur active disturbance, once the grid is disconnected, the voltage or frequency of the system will be unstable. If the system parameter exceeds the set thresholds and it is considered to have an islanding.

(1) the positive feedback islanding detection voltage is dynamically modify the amplitude of the reference current, once found common node voltage deviates from the original value, the given current modifies magnitude at the same direction. To make voltage change in the original direction further enhance until beyond the voltage protection threshold, then it is considered to have an islanding. The principle of the method is simple, easily achieved, but the output power quality will be reduced, at the same time the system transient response will be affected.

(2) active power disturbance islanding detection method is to control periodically output active power of PV system, once the grid is disconnected, the disturbance will cause the voltage at the common node exceeds the set threshold, thereby confirming there occurred an islanding This test method affected not by load properties. But periodic interference is caused by active power waste and injection into the grid energy fluctuations, and for the presence of multiple distributed power generation system, the role of the average effect of the impact of interference single power generation system on the overall line is not obvious.

Other active islanding detection method, there are many, such as sliding mode frequency drift method, reactive power perturbation method, the frequency drift assay and so on.

\section{Active Anti-Islanding Detection Frequency Drift}

Active frequency drift method is a reference frequency or phase of the current in each period are offset a little, then the frequency or phase of output current also will drift. When the grid is normal, the frequency of output current be re-aligned frequency of the grid voltage per cycle; when the grid has been disconnected, the phase or frequency of the reference current and grid voltage is no longer the same, since the reference value continuously offset the changing the frequency of the output current, frequency protection until beyond the threshold, then considered an island occurred. The method has simple, no extra hardware, etc., multi-machine grid systems as effective. Shown in figure 1 , by every period in the system and added a current reference dead time, so that the formation of the phase difference $t_{Z}$ between current and grid voltage. In the normal, the frequency of current remains unchanged since the grid voltage frequency does not change; when the formation of islands, the frequency of grid voltage will vary with the frequency of the current varies.According to anti-islanding detection criteria, when the grid voltage the frequency offset reaches a certain threshold can be considered an island occurred. 


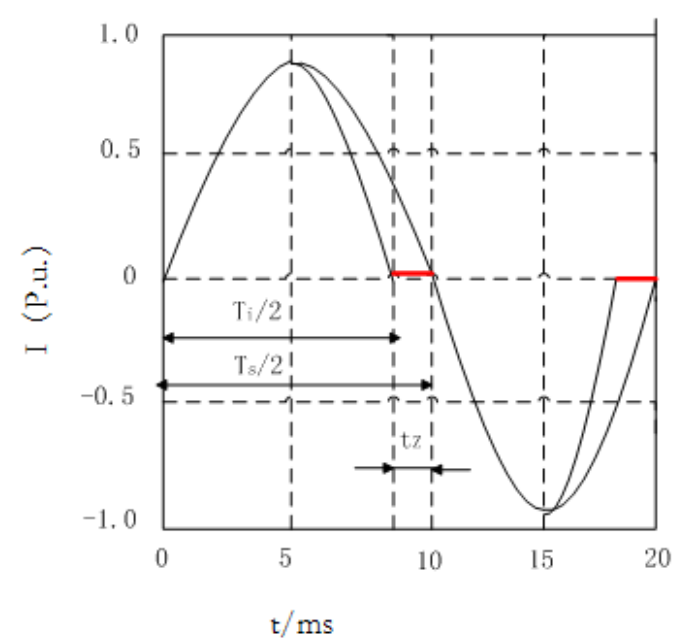

Fig 1.AFD islanding detection method the output current waveform

According to Figure 1. Important parameters derived active frequency drift - chopping fraction, cf.

$$
\Delta \phi=\frac{w t_{z}}{2}=\frac{2 \pi t_{z}}{2 * T_{1}}=\frac{\pi c f}{2}
$$

By Eq. 1 shows that if cf value is too small, to overflow frequency protection threshold prolonged, rapid detection methods can not be achieved; if cf value is too large, and Grid current distortion becomes large, and the harmonic wave content will increase, seriously affecting the quality of the system power quality. So how to choose cf value is the active frequency drift key issues law. In order to reduce the frequency drift active islanding detection blind spots and speed up response time, offset the use of positive feedback, and can accelerate the drift cf, shorten the time required frequency protection threshold time. Expression as follows:

$$
c f_{k}=c f_{k-1}+k_{f} F(\Delta f)
$$

\section{Based on variable active power active frequency drift anti-islanding detection}

By actual observation, when islanding occurs,not only frequency changes but also it often accompanied by active power changes. So adding variable active power into offset of active frequency drift anti-islanding detection, and time of anti-islanding detection can be shortened.

Output current frequency of expression:

$$
f_{k}=f_{k-1}+\operatorname{sign}\left(f_{0}-f_{k-1}\right) \frac{k_{\mathrm{p}}\left|\Delta P_{k-1}\right|}{p_{\mathrm{ac}}} \Delta f_{C}+\frac{k_{f}}{f_{0}}\left(f_{0}-f_{k-1}\right)
$$

Where ${ }^{\Delta P_{k-1}}$ - two cycles of output power difference;

$$
\begin{aligned}
& \Delta f_{\mathrm{C}} \text { - The frequency offset of the active phase; } \\
& f_{k} \text { - Output current frequency; } \\
& P_{\mathrm{ac}} \text { - Injection of active power grid normal. }
\end{aligned}
$$

If the variationof the output active power is the larger, Which is the difference $\triangle \mathrm{P}_{\mathrm{k}-1}$ is the larger, frequency offset increased migration step that reaches the system frequency protection threshold. If a smaller difference $\triangle \mathrm{Pk}-1$, the original frequency offset plays a leading role in the offset frequency at the frequency offset change, and variable active power play a supporting role. To avoid non-resistive load offsets consistent with the load phase, resulting in test failure. Based on variable active power active frequency drift anti-islanding detection not only the active power of information, but also includes changes in the grid reference current Iref, therefore frequency offset can better reflect changes in the entire system. 
Shown in figure 1, islanding detection flowchart using based on variable active power active frequency drift anti-islanding detection.

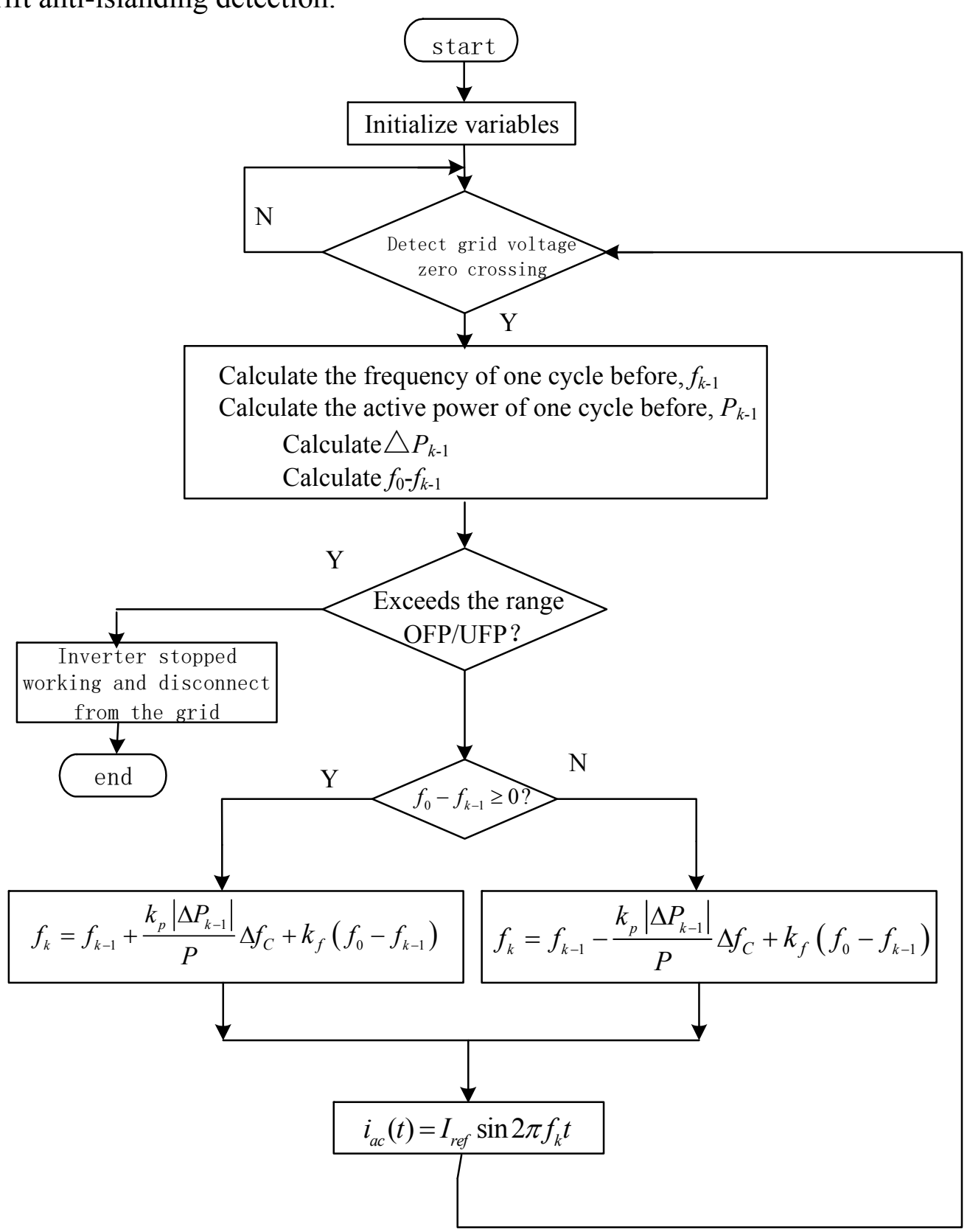

Fig.2 Based on Active Variable initiative frequency drift method flowchart

\section{The Simulation And Experimental Results}

In order to verify the effectiveness of islanding detection method proposed by Matlab / simlink simulation research.

Figure 3 is a photovoltaic grid islanding detection simulation AFD algorithm based on variable active power. The parameters of model are as follows: $\mathrm{DC} 380 \mathrm{~V}$, filter inductor $\mathrm{L}=8 \mathrm{mH}$ and filter capacitor $\mathrm{C}=10 \mathrm{PF}, \mathrm{R}=\mathrm{R} 1=5 \Omega$, $\mathrm{RMS}$ of ac voltage source is $220 \mathrm{Vand}$ frequency is $50 \mathrm{~Hz}$. 


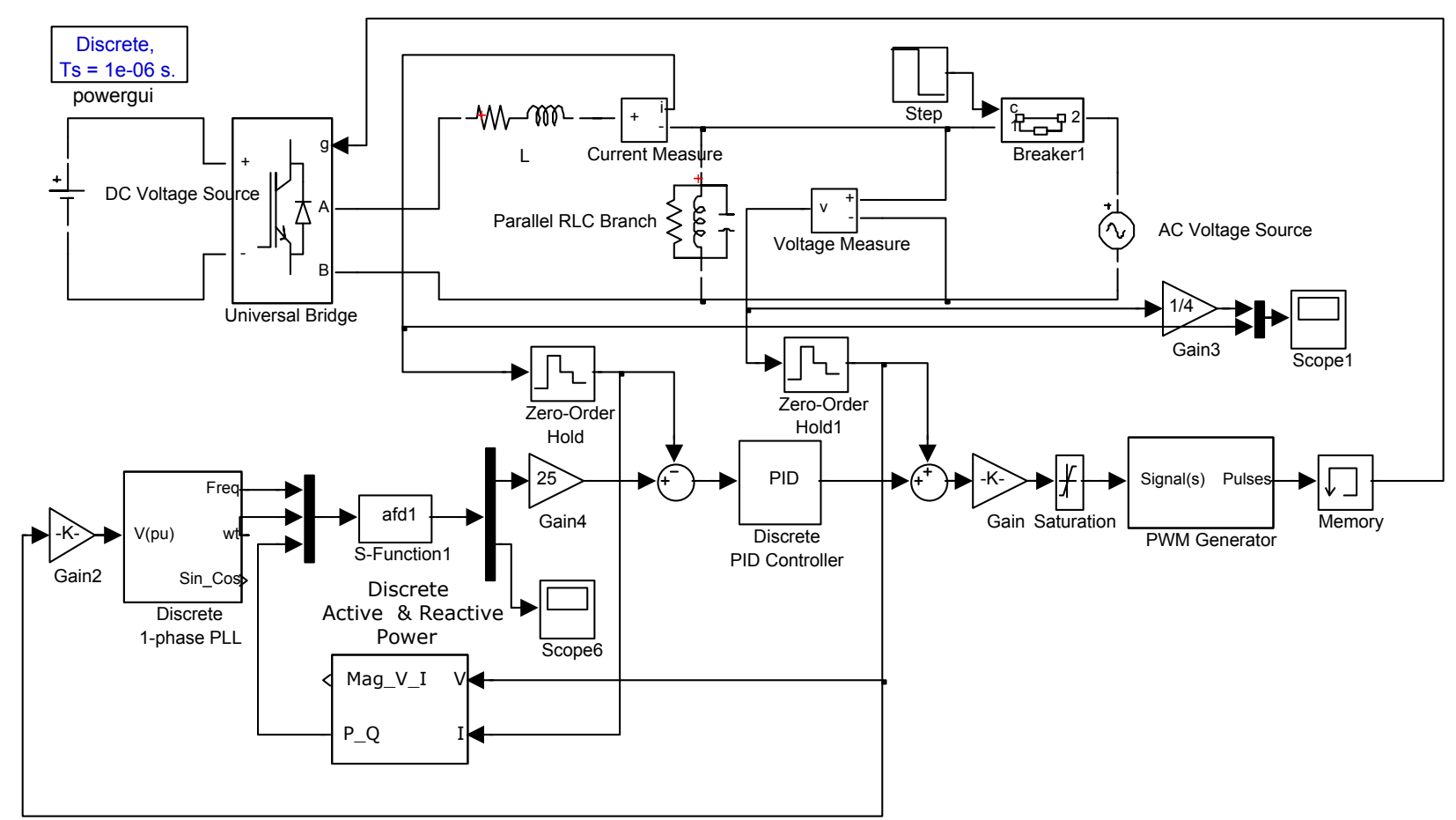

Fig.3 Based on Active Variable initiative frequency drift method simulation

The simulation is based structure figure 3 and islanding detection flowchart in figure 2, and Figure 4 shows output waveforms of voltage and current in purely resistive loads, that is the use of positive feedback active frequency drift method. change in frequency corresponding shown in figure 6 . From figure 3 shows that at the time of $0.06 \mathrm{~s}$ off the grid, offset by the positive feedback effect, increasing the frequency deviation, around $140 \mathrm{~ms}$ output frequency reaches frequency protection threshold, detects islanding occurs, the PV system stops working. Changes off the grid frequency needs some time to achieve protection threshold, and the output current due to chopping reason slight distortion. To further reduce the detection time, the need to increase the amount of frequency drift coefficient $\mathrm{k}_{\mathrm{f}}$, However, large $\mathrm{k}_{\mathrm{f}}$ will further increase output current waveform distortion situation.

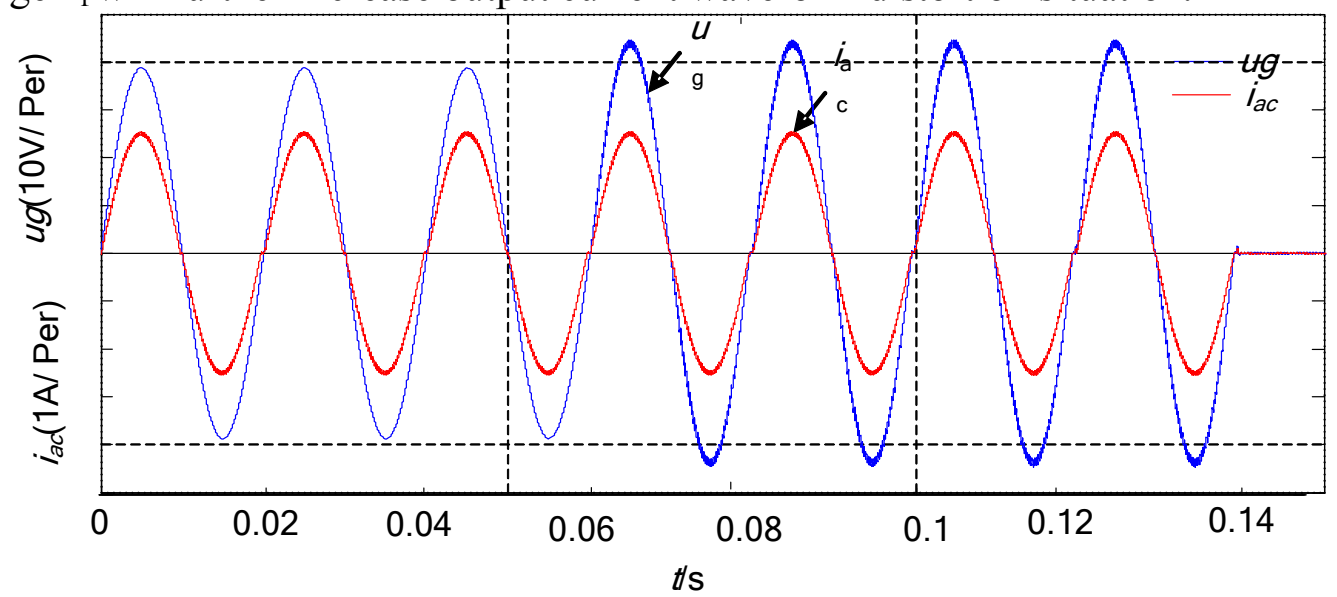

Fig.4 Output waveforms of AFD islanding detection with resistive load

Figure 5 shows a pure resistive load, simulation output voltage and current waveforms of based on variable active power active frequency drift anti-islanding detection, and change in frequency corresponding as shown in Figure 7 . From figure 3 it shows that at the time of $0.06 \mathrm{~s}$ off the grid, due to changes in the frequency deviation of the active power increases, accelerated the offset frequency. Around 120ms output frequency reaches frequency protection threshold is detected island phenomenon occurs, the PV system to stop working. Based on variable active power active frequency drift anti-islanding detection and positive feedback islanding detection method, compared to the islands used to detect the time about shortening $20 \mathrm{~ms}$, and the output current during normal operation waveform distortion little change. 


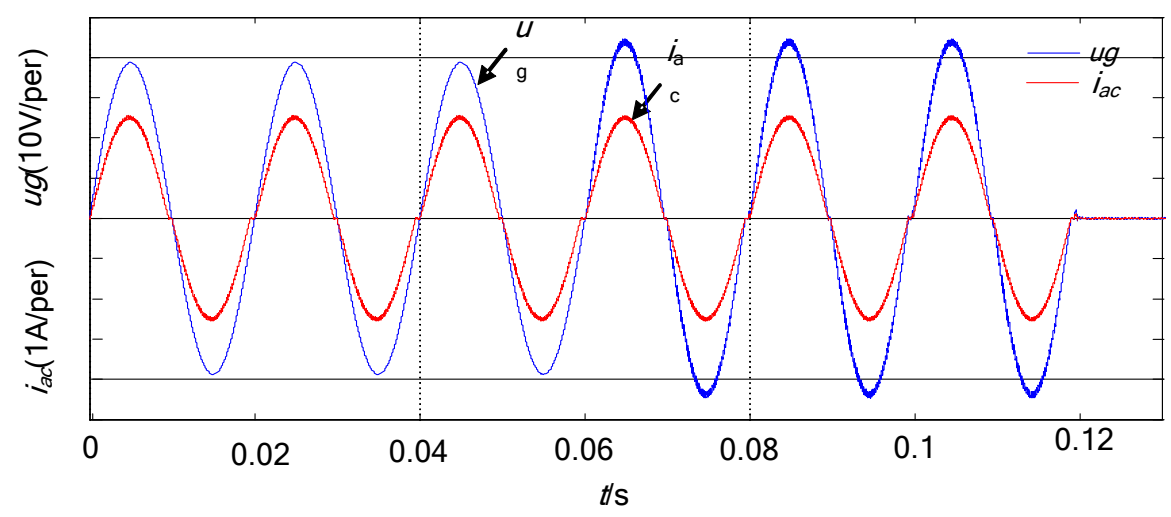

Fig.5 Output waveforms of AFD islanding detection on variable power with resistive load

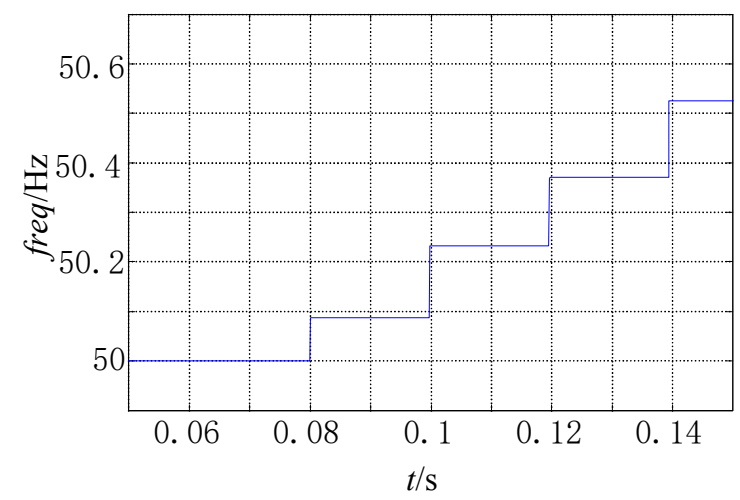

Fig.6 Voltage frequency of of AFD islanding detection

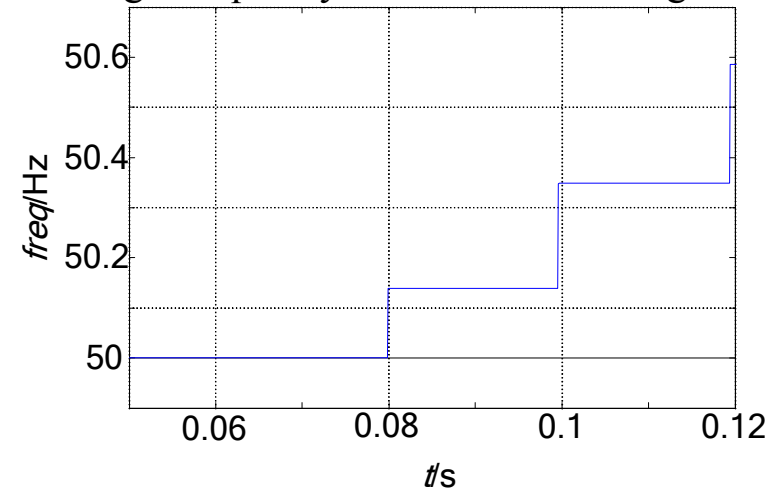

Fig.7 Voltage frequency of of AFD islanding detection on variable power

Figure 8 shows the experiment results based on variable active power active frequency drift anti-islanding detection, the figure shows a common point voltage and grid current waveform. When the grid is disconnected, the frequency drift due to changes in the active power increase, after about three cycles to detect the frequency exceeds the protection threshold, the system stops working.
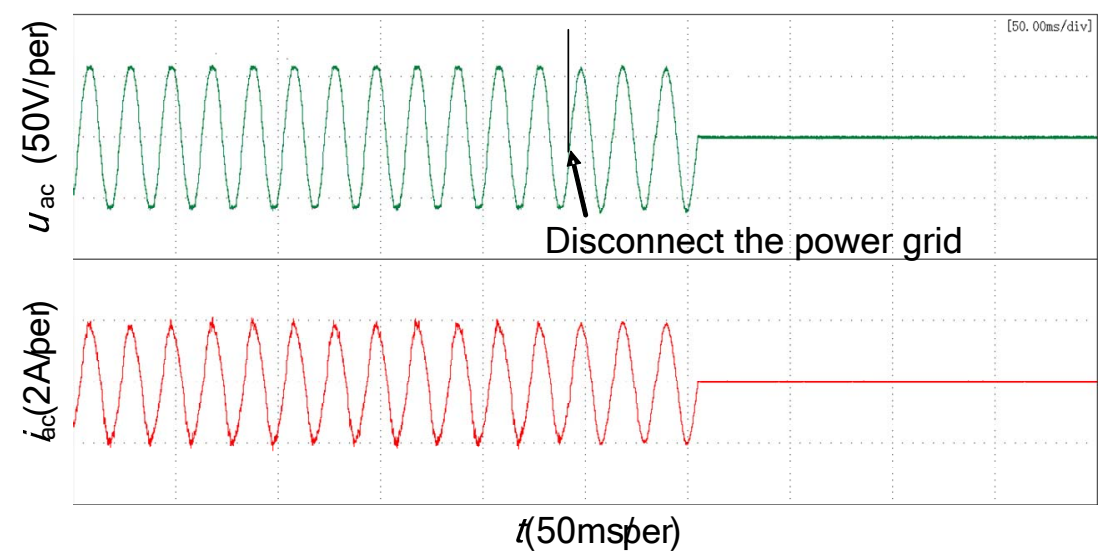

Fig.8 Experimental results of AFD on variable power 


\section{Conclusion}

In this paper, based on variable active power active frequency drift anti-islanding detection is proposed, by adding variable active power into offset. It will increase frequency of positive feedback of the voltage. Comparing based on variable active power active frequency drift anti-islanding detection method and positive feedback islanding detection method, proposed methods not only improve detected speed of anti-islanding detection, but also to ensure have better power quality that the injected into the grid

\section{Reference}

[1] Zhang Xueguang, Wang Rui, Liu Xinlong, Xu Dianguo, Improved Active Frequency Drift Anti-islanding Algorithm, Automation of Electric Power Systems, 2012(14), pp. 200-201

[2]Gao Zhi-qiang, JI Yan-chao, WANG Jian-ze, TAN Guang-hui, Improved control of Z-source PV inverter with inductive load, Electric Power Automation Equipment, 2011(7), pp. 27-30.

[3] Gao Zhi-qiang, WANG Jian-ze,JI Yan-chao, A fast method of maximum power point tracking for PV, Power System Protection and Control,, 2012(8), pp.105-109.

[4]H.Karimi, A. Yazdani, R. Iravani. Negative-Sequence Current Injection for Fast Islanding Detection of a Distributed Resource Unit[J]. IEEE Trans. on Power Electronics. 2008, 23(1): 298-307.)

[5] V. John, Z.H. Ye, A. Kolwalkar. Investigation of anti-islanding protection of power converter based distributed generators using frequency domain analysis[J]. IEEE Trans. on Power Electronics. 2004, 19(5): 1177-1183. 